\title{
Thickness Measurements With Electron Energy Loss Spectroscopy
}

\author{
K. IAKOUBOVSKII, ${ }^{1 *}$ K. MITSUISHI, ${ }^{1}$ Y. NAKAYAMA, ${ }^{2}$ AND K. FURUYA ${ }^{2}$ \\ ${ }^{1}$ Quantum Dot Research Center, National Institute for Materials Science, Tsukuba 305-0005, Japan \\ ${ }^{2}$ High Voltage Microscopy Station, National Institute for Materials Science, Tsukuba 305-0005, Japan
}

KEY WORDS TEM; EELS; inelastic scattering; mean free path

\begin{abstract}
Measurements of thickness using electron energy loss spectroscopy (EELS) are revised. Absolute thickness values can be quickly and accurately determined with the KramersKronig sum method. The EELS data analysis is even much easier with the log-ratio method, however, absolute calibration of this method requires knowledge of the mean free path of inelastic electron scattering $\lambda$. The latter has been measured here in a wide range of solids and a scaling law $\lambda \sim$ $\rho^{-0.3}$ versus mass density $\rho$ has been revealed. EELS measurements critically depend on the excitation and collection angles. This dependence has been studied experimentally and theoretically and an efficient model has been formulated. Microsc. Res. Tech. 71:626-631, $2008 . \quad$ o 2008 Wiley-Liss, Inc.
\end{abstract}

\section{INTRODUCTION}

Thickness measurements in transmission electron microscopy (TEM) are important as they bring quantitative nanoscale information. Contrary to lateral $x-y$ mapping, which can be easily performed with high precision, thickness measurements concern $z$ direction of the propagating electron beam, which is difficult to monitor in TEM. Consequently, the relevant measurement techniques are often complex and indirect, and they require modeling and thus are prone to skepticism. For example, most popular methods for thickness measurements in TEM use convergent-beam electron diffraction (CBED) and electron energy loss spectroscopy (EELS). The former technique is relatively difficult technically, as it requires accurate sample alignment and tedious data analysis. It has also rather narrow application range-measured region must be a homogeneous single crystal of thickness $(\sim 150-300 \mathrm{~nm})$. However, the analyzed phenomenon-interference fringes - can be intuitively related to the sample thickness, and, thus, the technique is widely considered as highly "reliable." On the contrary, EELS methods are much easier and faster both in acquisition and analysis. They do not require specific crystalline structure and work in a wide thickness range ( $15-500 \mathrm{~nm}$, see e.g., Mitchell, 2006; Egerton and Chen, 1987). However, the resulting energy-loss spectra are rather indirectly related to the thickness, and thus the technique has lower trust among many electron microscopists. This skepticism is partially justified as the modeling of EELS thickness measurements have indeed had some obvious flaws discussed below. In this article, we attempt to bring more clarity into the relevant experiment and interpretation, thus hoping to improve the popularity of the EELS methods.

Thickness measurements by EELS rely on the analysis of an experimental spectrum. The latter includes a strong peak at zero-loss energy, therefore called zeroloss peak (ZLP), and a significantly weaker and broader spectrum of inelastically scattered electrons (see Fig. 1). The spectral shape depends on the experimental conditions such as the microscope voltage $E_{0}$, conver- gence semiangle of the excitation beam $\alpha$, collection semiangle $\beta$, and the structure of the irradiated sample. The analysis is commonly performed with either the Kramers-Kronig sum model, hereafter referred to as KK model, or the log-ratio model. The associated thickness calculation is fast, can be performed in "real time," and is implemented in the popular program Digital Micrograph by Gatan. The associated modeling is mostly by Egerton (Egerton, 1996; Egerton and Chen, 1987; Malis et al., 1988; Yang and Egerton, 1995) and is discussed below. A word of caution should be said however to the Digital Micrograph users: simple trial tests reveal that some of the associated routines, such as extraction of the real and imaginary parts of the dielectric constant and the correction for the convergence semiangle, produce results, which do contradict to the experiment (Egerton, 1996; Malis et al., 1988).

\section{Kramers-Kronig Sum Model}

This model analyses the single scattering distribution $S(E)$, which is obtained from the EEL spectrum $J(E)$ by removing the ZLP and the contribution of plural inelastic scattering. The removal procedure is straightforward (Egerton, 1996) and is performed automatically, for example, with the Digital Micrograph routines. The thickness $t$ is deduced with the following formulas:

$$
t=\frac{4 a_{0} F E_{0}}{\left(1-1 / n^{2}\right) I_{0}} \int \frac{S(E) \mathrm{dE}}{E \ln \left(1+\beta^{2} / \theta_{\mathrm{E}}^{2}\right)}, \quad F=\frac{\left(1+E_{0} / 1022\right)}{\left(1+E_{0} / 511\right)^{2}}
$$

where $I_{0}$ is the integrated ZLP intensity, $F$ is a relativistic factor, $a_{0}$ is the Bohr radius, $\theta$ is scattering angle,

\footnotetext{
*Correspondence to: K. Iakoubovskii, Quantum Dot Research Center, National Institute for Materials Science, 3-13 Sakura, Tsukuba 305-0005, Japan.

E-mail: iakoubovskii.konstantin@nims.go.jp

Received 5 January 2008; accepted in revised form 29 February 2008

DOI 10.1002/jemt.20597

Published online 2 May 2008 in Wiley InterScience (www.interscience.wiley. com).
} 


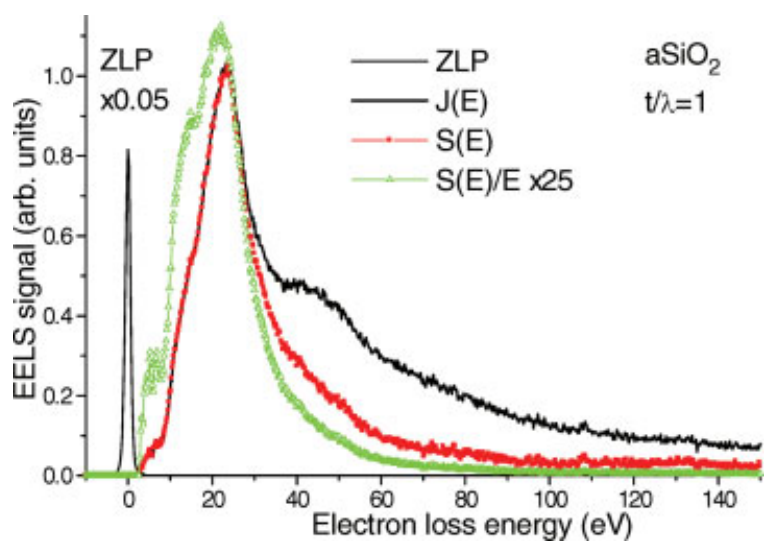

Fig. 1. Typical EEL spectra from amorphous $\mathrm{SiO}_{2} . J(E)$ is the asmeasured spectrum (acquisition time $50 \mathrm{~ms}$ ) with the ZLP multiplied by 0.05 . Removal of the ZLP and plural scattering contributions results in spectrum $S(E)$. Division of $S(E)$ by $E$, as appropriate for the Kramers-Kronig analysis, improves the $S / N$ ratio and produces a more energy-localized spectrum. [Color figure can be viewed in the online issue, which is available at www.interscience.wiley.com.]

$\theta_{E} \cong E /\left(2 F E_{0}\right)$ is the characteristic angle of inelastic scattering corresponding to an energy loss $E$; the microscope voltage $E_{0}$ is in kilovolt units. Division of $S(E)$ by $E$ in Eq. (1) makes the spectrum more localized in energy scale; it therefore improves the signal-tonoise ratio at high energies and reduces the required spectral window (see Fig. 1).

The appearance of refractive index $n$ in Eq. (1) is important and deserves several comments:

- The model assumes static $n$ value, that is, $n(E=0)$. However, in practice, $n(E \sim 1-2 \mathrm{eV})$ should be used for a typical high-voltage microscope because the finite spread of the ZLP masks the low-energy region.

- Equation (1) could be rather sensitive to the $n$ value. For example, until the very recent 1.7 version, the Digital Micrograph program rounded the $n$ value to 0.1 that resulted in $\Delta n$ uncertainty of $\sim 0.05$. This produced the thickness error of only $\sim 0.3 \%$ for silicon $(n=3.42)$, but the error increased to $\sim 6 \%$ for $\mathrm{SiO}_{2}(n=1.5)$.

- If thickness is known from independent measurements then Eq. (1) can be reversed to determine the refractive index with the high spatial resolution of TEM.

The usage of the refractive index is probably the main disadvantage of the KK method as its accurate value is yet uncertain for many materials, especially in their nanoscaled forms.

\section{Log-Ratio Model}

The apparent simplicity of this model makes it one of the most popular tools of thickness measurements by EELS (see e.g., Crozier, 1990; Egerton and Chen, 1987; Lee et al., 2002; Malis et al., 1988; Mitchell, 2006; Nakafuji et al., 2001; Ohshima et al., 2004; Yang and Egerton, 1995). Two modifications of this method can be distinguished, "relative" and "absolute."

The former uses only a very reasonable assumption of independent nature of scattering events and results

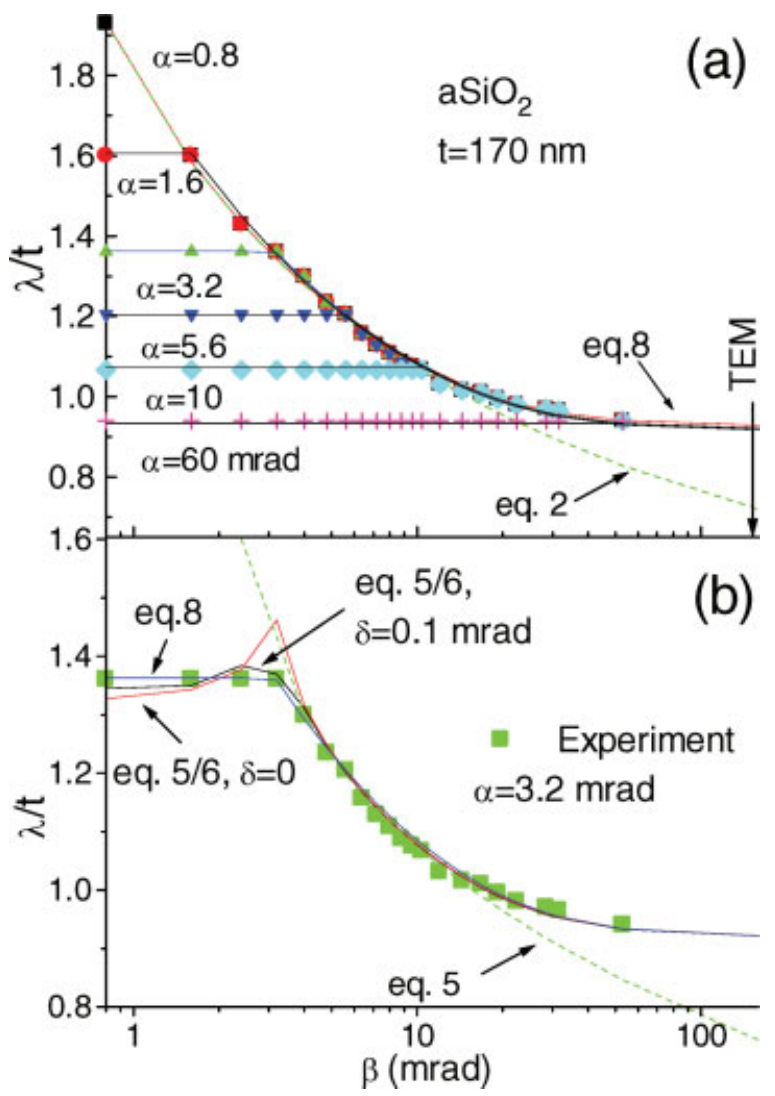

Fig. 2. Scatter graphs present experimental dependences of the relative mean free path of inelastic scattering, measured from amorphous $\mathrm{SiO}_{2}\left(t=170 \mathrm{~nm}, E_{0}=200 \mathrm{kV}\right.$, STEM imaging mode), on the collection semiangle $\beta$ for several indicated values of the convergence semiangle $\alpha$. Lines show simulation with Eqs. (2) and (8), as discussed in Angular dependence of the inelastic mean free path section.. The arrow with symbol TEM indicates a typical TEM collection angle, for which a significant deviation is observed between the experiment and the commonly used angular correction. [Color figure can be viewed in the online issue, which is available at www.interscience.wiley.com.]

in a simple expression $t / \lambda=\ln \left(I / I_{0}\right)=\int J(E) \mathrm{d} E / I_{0}$, where $I$ the area under the whole EEL spectrum. With this method, easy and reliable, though relative, thickness measurements can be performed.

Absolute measurements with the log-ratio method require knowledge of the mean free path of inelastic electron scattering $\lambda$. This fundamental quantity is difficult to calculate with sufficient accuracy, and the experimental values are scarce and, as mentioned earlier, strongly depend on the material and the measurement conditions. This dependence has been approximated (Egerton and Chen, 1987) using Eq. (1) as follows:

$$
\begin{gathered}
\lambda=\frac{t}{\ln \left(I / I_{0}\right)}=\frac{4 a_{0} F E_{0}}{\left(1-1 / n^{2}\right) I_{0} \ln \left(I / I_{0}\right)} \int \frac{S(E) \mathrm{dE}}{E \ln \left(1+\beta^{2} / \theta_{\mathrm{E}}^{2}\right)} \\
\approx \frac{106 F E_{0}}{E_{\mathrm{m}} \ln \left(2 E_{0} \beta / E_{\mathrm{m}}\right)}
\end{gathered}
$$

The average energy loss $E_{\mathrm{m}}$, which summarizes the material properties, is only known for a limited range 
of solids (Egerton, 1996, p. 304). Therefore, an experimental scaling law has been proposed as

$$
\begin{array}{r}
E_{\mathrm{m}}=\frac{\left(1-1 / n^{2}\right) I_{0} \ln \left(I / I_{0}\right)}{\int S(E) / E \mathrm{dE}} \cong \frac{\left(1-1 / n^{2}\right) \int J(\mathrm{E}) \mathrm{dE}}{\int S(\mathrm{E}) / \mathrm{EdE}} \\
=7.6 \mathrm{Z}_{\mathrm{ef}}^{0.36}, \quad Z_{\mathrm{ef}}=\sum_{i} \frac{f_{i} Z_{i}^{1.3}}{f_{i} Z_{i}^{0.3}}
\end{array}
$$

Here, the effective atomic number $Z_{\text {ef }}$ is expressed via the number $f_{i}$ of atoms with atomic number $Z_{i}$.

\section{Inconsistencies of the Kramers-Kronig and Log-Ratio Models}

A closer look at the data (Egerton, 1996, p. 304; Mitchell et al., 1988) behind the scaling law of Eq. (3) reveals significant experimental scatter. Another set of data (Crozier, 1990) has been simulated with a rather different $E_{\mathrm{m}} \sim Z^{0.57}$ dependence. The standard deviation was much smaller than in the data used for Eq. (3), however only six materials have been used in the analysis.

Equation (3) expresses all properties of a solid merely by an atomic number of its constituents. Intuitively, one could also expect $\lambda$ to be proportional to the number of atoms per unit volume $\rho / A$, where $\rho$ is mass density and $A$ is the atomic mass.

The $\lambda(\beta)$ dependence of Eqs. (1) and (2) agrees with the experiment only for small collection angles $(\beta<20$ mrad). A saturation, not included in the model, is observed experimentally for larger $\beta$ (see e.g., Crozier, 1990; Lee et al., 2002; Malis et al., 1988; Mitchell, 2006; Nakafuji et al., 2001; Ohshima et al., 2004; and Fig. 2 discussed below). Consequently, for a typical TEM collection angle of $160 \mathrm{mrad}$, Eqs. (1) and (2) underestimate thickness by $\sim 20 \%$. It is important to note that this tendency is specific not only to thickness measurements, but to many other models of electron scattering where a simple Lorentzian angular distribution is assumed. This deviation is well known, but nevertheless remains uncorrected. It is associated (Egerton, 1996) with a faster than the Lorentzian $\left(1 / \theta^{2}\right)$ decrease of the scattering cross section at large scattering angles or, in other words, with "breakdown of a dipole approximation."

At the time of developing the $\mathrm{KK}$ and log-ratio methods, most TEM microscopes used nearly parallel excitation beam. However, nowadays, for a number of reasons such as availability of aberration correctors, scanning transmission electron microscopes (STEMs) are increasingly popular. In STEM, values of $\alpha$ and $\beta$ are comparable and thus the convergence angle correction is required. Such correction has been suggested by Egerton (1996, p. 284) and implemented in the Digital Micrograph, but unfortunately, it does not agree with the experiment (Malis et al., 1988).

In this article, we correct the above-mentioned drawbacks and propose improvements to the Eqs. (1) and (2). Those corrections include a simple and accurate scaling law of the total inelastic mean free path with material properties as $\lambda \sim \rho^{-0.3}$. In addition, a model is proposed accounting for the convergence angle dependence of inelastic electron scattering.

\section{EXPERIMENTAL}

EELS measurements were performed with a Jeol high-vacuum aberration-corrected 2500SES STEM microscope $(200 \mathrm{kV})$ equipped with an Enfina EEL spectrometer. Under electron irradiation, TEM samples often rapidly accumulate carbon-related contamination layers that results in the overestimated thicknesses. Using high vacuum $\left(\sim 10^{-6} \mathrm{~Pa}\right)$ reduced contamination in this study. The spherical aberration corrector allowed to apply a wide range of excitation angles $\alpha$ without significant angular distortions and to achieve small beam size. Small beam size and utilization of the imaging mode reduced the inhomogeneityrelated problems (thickness variation within the probed area).

It should be noted that most measurements of this paper aim at deducing the values of inelastic mean free path $\lambda$ as function of material structure and measurement geometry. Those variations are rather small, and therefore their measurement requires improved accuracy. This was achieved in the following way: All measurements were performed sequentially, that is, without major microscope realignment in between. Apart from the angular dependences of Figure 2, they were performed at nominally same microscope settings. Excitation semiangle was fixed at $\alpha=20 \mathrm{mrad}$ and collection semiangle at $\beta=5 \mathrm{mrad}$, that is, $\left(\theta_{E} \ll \beta<\alpha\right)$. This reduced the angular sensitivity of the measurements (see Fig. 2) and the small-angle misalignment problems.

EEL spectra were measured in a $300 \mathrm{eV}$ window, which contained virtually all the spectral intensity. Moderately thin sample regions have been chosen $(t / \lambda$ $\sim 1$ or $70<t<180 \mathrm{~nm}$ ) to reduce the surface plasmon contributions, increasing at small thicknesses, and plural scattering at large thicknesses.

To measure $\lambda$ by EELS, one needs to know the sample thickness. For many studied samples, it was measured with the KK method whose reliability has been demonstrated already (Egerton and Chen, 1987; Yang and Egerton, 1995). However, to further test this method, the following procedure has been applied. It consisted of specific sample preparation (1) and measurement procedure (2).

Several layered structures $\mathrm{Si} / \mathrm{aSiO}_{2} / \mathrm{Ta} / \mathrm{CoO} / \mathrm{Pt} / \mathrm{aC}$, $\mathrm{Si} / \mathrm{aSiO}_{2} / \mathrm{Ta} / \mathrm{Pt} / \mathrm{NiO} / \mathrm{Al}, \quad \mathrm{Si} / \mathrm{SiO}_{2} /$ epoxy/Au/Si/aC, $\mathrm{Si} /$ $\mathrm{aSiO}_{2} / \mathrm{Cr} / \mathrm{Pt} / \mathrm{W}$, and $\mathrm{Si} /$ diamond have been prepared using conventional techniques of magnetron sputtering, thermal evaporation, chemical vapor deposition, and epoxy gluing. Note that here and further letter "a" before $\mathrm{SiO}_{2}$ or $\mathrm{C}$ corresponds to the amorphous phase. Cross-sectional TEM samples were prepared from those structures by focused ion beam cutting $\left(\mathrm{Ga}^{+}, 5-\right.$ $30 \mathrm{keV}$ ). The cutting was performed normal to the layer surface, that is, through all the layers. Ion-beam cutting allowed to produce samples with uniform, almost constant, thickness profile and to avoid sharp thickness variation at the interfaces. The latter is known as "differential thinning" and is common for most other sample preparation techniques.

Measurements on those layered structures were performed in the following steps:

- Thickness of the Si layer has been measured by CBED. 


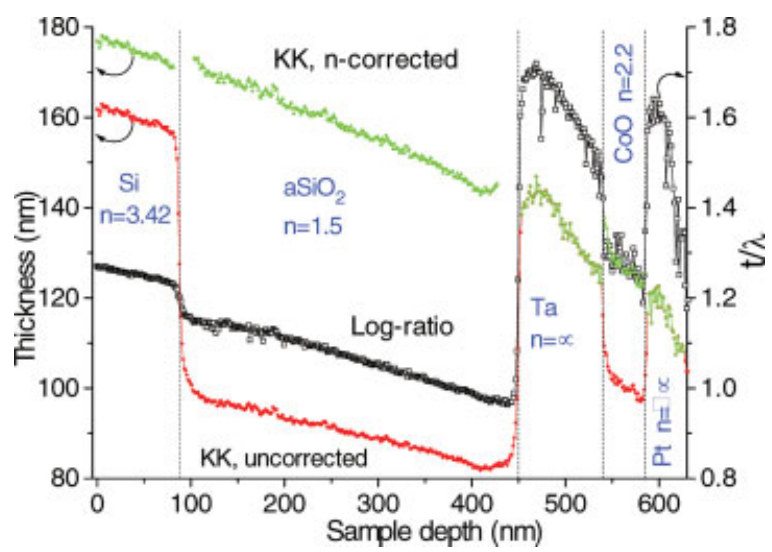

Fig. 3. A summary of the analysis of a line scan profile from the $\mathrm{Si} / \mathrm{aSiO}_{2} / \mathrm{Ta} / \mathrm{CoO} / \mathrm{Pt} / \mathrm{aC}$ sample. The bottom curve ( $\mathrm{Si}$ area, black open squares) shows $t / \lambda$ values obtained with the relative log-ratio model. Middle and top curves (solid symbols) correspond to the KK model applied without and with refractive index correction $\left[1-1 / n^{2}\right.$ term in Eq. (2)]. A smooth thickness profile is obtained with the corrected KK model, as expected for an FIB-prepared TEM sample. [Color figure can be viewed in the online issue, which is available at www. interscience.wiley.com.]

- EEL spectra were acquired at (500 spatial points along a straight line running through all the layers.

- The EELS data was analyzed with a "relative" logratio model yielding $\lambda / t$ line profile (see Fig. 3). Smooth thickness profile allowed to obtain relative $\lambda$ values referenced to one sample material. Crystalline $\mathrm{Si}$ has been chosen as such reference-its $\lambda$ value for accelerating voltage $200 \mathrm{kV}$ has been measured by several groups (see e.g., Jin, 2004; Krivanek et al., 1990; Lee et al., 2002; Mitchel, 2006), and a good agreement has been achieved with the angular-saturated $\lambda$ value of $\sim 145 \mathrm{~nm}$, most accurately measured by Jin (2004).

- The relative $\lambda$ values have been converted to the absolute ones using the CBED results for Si. The obtained $\lambda$ value for $\mathrm{Si} 142 \pm 5 \mathrm{~nm}$ was consistent with the previous measurements.

- The KK model has been applied to the EELS data. Consistent results, both in the absolute values and in their line profiles across the layered structures (see Fig. 3), were obtained thus additionally confirming the validity of the Kramers-Kronig model.

Therefore, for $\lambda$ measurements in other materials, homogeneous samples have been chosen. Their thickness was measured by EELS using the KK analysis and then corresponding $\lambda$ values were deduced with the relative $\log$-ratio model, that is, equation $t / \lambda=\ln \left(I / I_{0}\right)$. The absolute log-ratio method [Eq. (2)] has not been used in the analysis; instead, its corrected equations have been developed in section Angular Dependence of the Inelastic Mean Free Path.

\section{RESULTS}

Figure 2a shows the dependences of $1 / \ln \left(I / I_{0}\right)=\lambda / t$ on the collection semiangle $\beta$ for several values of the collection semiangle $\alpha$. Measurements were performed from the same, subnm sized spot of a homogeneous

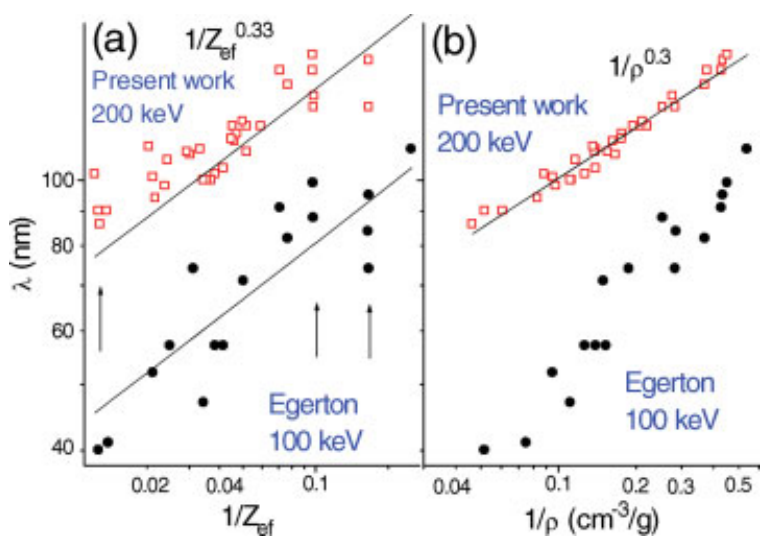

Fig. 4. Experimental values of inelastic mean free path $\lambda$ obtained for a wide range of materials in the present (top open squares) and previous (bottom solid circles, Egerton 1986, p. 304) studies. The data correspond to angular-saturated values. They are presented as double-logarithmic plots versus the inverse effective atomic number $1 / Z_{\text {ef }}$ (panel a) and inverse mass density $1 / \rho(\mathbf{b})$. A significantly better scaling with $1 / \rho$ as $\lambda \sim \rho^{-0.3}$ is revealed. [Color figure can be viewed in the online issue, which is available at www.interscience.wiley.com.]

$\mathrm{SiO}_{2}$ foil, i.e., thickness could be considered constant and the measured dependences associated with $\lambda$ variations only. Lines in Figure 2 present simulation which is discussed in Angular dependence of the inelastic mean free path section. Diffraction effects are especially important in angular-dependent measurements as they can modulate the EELS intensity (Mitchell, 2006; Nakafuji et al., 2001) and thus result in overestimation of $\lambda$ by up to $25 \%$ (Yang and Egerton, 1995). Therefore, measurements summarized in Figure 2 were performed on amorphous $\mathrm{SiO}_{2}$. Similar $\lambda(\alpha, \beta)$ dependences have been reported (Malis et al., 1988) for three values of $\alpha$ but have not been explained by modeling. It is worthwhile to note that very similar curves were obtained in this work using either log-ratio or KK model, and thus the developed below angular correction should be applied to both methods.

Figure 3 summarizes the analysis of a line scan profile from the $\mathrm{Si} / \mathrm{aSiO}_{2} / \mathrm{Ta} / \mathrm{CoO} / \mathrm{Pt} / \mathrm{aC}$ sample. The bottom black open squares ( $\mathrm{Si}$ area) represent $t / \lambda$ values obtained with the relative log-ratio model. Middle and top curves (solid symbols) correspond to the KK model applied without and with refractive index correction $\left(1-1 / n^{2}\right.$ term in [Eq. (2)]). Thin interfacial regions have been excluded. Core-loss EELS measurements revealed that in those regions some material interdiffusion occurred making uncertain the $n$ and $\lambda$ values. The utilized refractive indices are copied into the Figure 3 , and they agree well with the published values. A better signal-to-noise ratio is obtained with the KK than with the log-ratio model reflecting the mentioned in the introduction advantages of the $J(E) / E$ function over $J(E)$. The thickness of the Si layer was independently measured by CBED as $175 \pm 5 \mathrm{~nm}$. The top curve reveals that refractive index correction produces smooth thickness profile, as expected for an FIB-prepared sample, and the thickness values for the Si layer are consistent with the CBED results. Using those values, $\lambda(\mathrm{Si})$ was calculated as $142 \pm 5 \mathrm{~nm}$, in agreement 
with the previous reports (Jin, 2004; Lee et al., 2002; Mitchel, 2006).

Measurements on two other layered structures yielded similar results and confirmed the validity and accuracy of the KK model for thickness measurements. Using a combination of the $\mathrm{KK}$ and relative log-ratio analysis, the $\lambda$ values for a wide range of materials (Ag, $\mathrm{Al}, \mathrm{Al}_{2} \mathrm{O}_{3}, \mathrm{Au}$, Ac, diamond, $\mathrm{CoO}, \mathrm{Cr}, \mathrm{Cu}, \mathrm{Fe}, \mathrm{GaN}$, In, $\mathrm{MgO}, \mathrm{Mo}, \mathrm{Nb}, \mathrm{Ni}, \mathrm{Pb}, \mathrm{Pd}, \mathrm{Pt}, \mathrm{Si}, \mathrm{aSiO}_{2}, \mathrm{cSiO}_{2}$, $\mathrm{SrTiO}_{3}$, Ta, Pt, V, W, Zn, ZnO, $\mathrm{ZrO}_{2}$ ) have been measured and plotted in Figure 4. Data of Egerton (1996 p. 304) for $\mathrm{Ag}, \mathrm{Al}, \mathrm{Al}_{2} \mathrm{O}_{3}, \mathrm{Au}, \mathrm{Be}, \mathrm{BN}, \mathrm{aC}, \mathrm{Cr}, \mathrm{Cu}$, diamond, Fe, GaAs, Hf, NiO, Si, $\mathrm{aSiO}_{2}, \mathrm{Zr}$ are also presented for comparison. Those values are smaller than ours because of smaller electron accelerating voltage $(100 \mathrm{kV}$ vs. $200 \mathrm{kV})$. Figure 4 presents these two sets of data in double logarithmic plots versus $1 / Z_{\text {ef }}$ (panel a) and $1 / \rho(b)$.

\section{ANALYSIS}

\section{Material Scaling of Mean Free Path}

Figure 4 reveals that plotting the same data versus $1 / \rho$ rather than versus $1 / Z_{\text {ef }}$ significantly reduces the scatter. This is especially obvious for three sets of materials marked by arrows in panel a: $\mathrm{Hf}, \mathrm{Ta}, \mathrm{Pt}, \mathrm{Pb}, \mathrm{Au}$; $\mathrm{aC}, \mathrm{BN}$, diamond; and $\mathrm{MgO}, \mathrm{Al}_{2} \mathrm{O}_{3}, \mathrm{aSiO}_{2}$, and $\mathrm{cSiO}_{2}$. They have almost identical $Z_{\text {ef }}$, but rather different $\lambda$ and $\rho$ values, and surprisingly, those $\lambda$ and $\rho$ values exhibit a simple $\lambda \sim \rho^{-0.3}$ correlation. Therefore, we propose to use this scaling in the Eq. (3) instead of the previously adopted $\lambda \sim Z_{\mathrm{ef}}^{-0.36}$ dependence.

Atomic scattering models suggest $\lambda$ to be proportional to the density of atoms $\rho / A$. However, attempts to scale the data of Figure 4 versus $\rho Z^{\mathrm{a}} / A$ failed for any value of the exponent $a$. This failure could be understood as inelastic electron scattering originates not only from atomic but also from electronic (plasmon) processes, and it will be discussed elsewhere (Iakoubovskii et al., 2008).

\section{Angular Dependence of the Inelastic Mean Free Path}

In this section, we develop a model for the angular dependences of $\lambda$ presented in Figure 2. We shall assume small angles such that $\sin \theta \cong \theta$ that adds negligible error for the angles $<150 \mathrm{mrad}$ considered. We shall be interested only in the angular dependence and thus omit most proportionality constants.

The inverse free path $1 / \lambda$ is proportional to the inelastic cross section $\sigma_{\text {in }}$, which can be obtained by angular integration of the partial cross section $d \sigma_{\text {in }} / \mathrm{d} \Omega$ as

$$
\sigma_{\text {in }}=\int \frac{\mathrm{d}^{2} \sigma_{\text {in }}}{\mathrm{d} \Omega} \mathrm{d} \Omega \propto \int \frac{|f(\mathrm{q})|^{2}}{\mathrm{q}^{4}} \mathrm{~d} \Omega
$$

Here $q^{2} \approx k_{0}^{2}\left(\theta^{2}+\theta_{\mathrm{E}}^{2}\right), \theta \gg \theta_{E}, k_{0}$, and $q$ are the amplitudes of the incident and scattering vectors, respectively; $f(q)$ is inelastic form factor, which modifies the Rutherford scattering $\sim 1 / q^{4}$. An assumption $|f(q)|^{2} \sim q^{2}$ leads to the Lorentzian angular distribution and thus the traditional $\ln \left(1+\beta^{2} / \theta^{2}\right)$ angular dependence. Here, we shall assume instead $|f(q)|^{2}=q^{2} /\left[1+q^{2} /\left(k_{0} \theta_{0}\right)^{2}\right]=$ $q^{2} /\left(1+\theta^{2} / \theta_{\mathrm{C}}^{2}\right)$, which results in a usual $\theta^{-2}$ dependence for small angles and a faster $\theta^{-4}$ decrease at larger angles.

The above equations are usually applied to the TEM mode, that is, a parallel incident beam $(\alpha=0)$. For nonzero $\alpha$, we calculate the scattering angle $\theta$ as a function of the convergence and collection angles $\theta_{1}$ and $\theta_{2}$ as $\theta^{2}$ $=\theta_{1}^{2}+\theta_{2}^{2}-2 \theta_{1} \theta_{2} \cos \theta$ and integrate Eq. (4) over the both solid angles as

$$
\begin{aligned}
\sigma_{\text {in }} & \propto \int \frac{\mathrm{d} \Omega_{1} \mathrm{~d} \Omega_{2}}{\left(\theta^{2}+\theta_{\mathrm{E}}^{2}\right)\left(1+\theta^{2} / \theta_{\mathrm{C}}^{2}\right)} \\
& \propto \int_{0}^{\alpha} \int_{0}^{\beta} \int_{0}^{2 \pi} \frac{1}{\theta^{2}+\theta_{\mathrm{E}}^{2}}-\frac{1}{\theta^{2}+\theta_{\mathrm{C}}^{2}} \theta_{1} \theta_{2} \mathrm{~d} \theta_{1} \mathrm{~d} \theta_{2} \mathrm{~d} \varphi \\
& \propto \sigma\left(\theta_{\mathrm{E}}\right)-\sigma\left(\theta_{\mathrm{C}}\right)
\end{aligned}
$$

where

$$
\begin{gathered}
\sigma\left(\theta_{\mathrm{E}}\right)=2 \alpha^{2} \ln \frac{\beta^{2}-\alpha^{2}+\theta_{\mathrm{E}}^{2}+\sqrt{\left(\alpha^{2}+\beta^{2}+\theta_{\mathrm{E}}^{2}\right)^{2}-4 \alpha^{2} \beta^{2}}}{2 \theta_{\mathrm{E}}^{2}} \\
+2 \beta^{2} \ln \frac{\alpha^{2}-\beta^{2}+\theta_{\mathrm{E}}^{2}+\sqrt{\left(\alpha^{2}+\beta^{2}+\theta_{\mathrm{E}}^{2}\right)^{2}-4 \alpha^{2} \beta^{2}}}{2 \theta_{\mathrm{E}}^{2}} \\
-\sqrt{\left.\left(\alpha^{2}+\beta^{2}+\theta_{\mathrm{E}}^{2}\right)^{2}-4 \alpha^{2} \beta^{2}\right)}+\left(\alpha^{2}+\beta^{2}+\theta_{\mathrm{E}}^{2}\right)
\end{gathered}
$$

and $\sigma\left(\theta_{\mathbf{C}}\right)$ is obtained by substituting $\theta_{\mathbf{C}}$ for $\theta_{E}$ in the above formula for $\sigma\left(\theta_{E}\right)$. The $\sigma_{\text {in }}$ needs to be normalized by the angular-dependent intensity of the electron beam $I_{0}$ falling on the EELS detector. Intuitively, we expect $I_{0} \sim \alpha^{2}$ for $\alpha<\beta$ and $I_{0} \sim \beta^{2}$ for $\alpha>\beta$, that is, $I_{0}$ $\sim \alpha^{2}+\beta^{2}-\left|\alpha^{2}-\beta^{2}\right|$. However, dividing Eq. (5) by this intensity function results in a significant deviation from the experiment for $\alpha \approx \beta$ (see curve "Eq. (5/6) $\delta=$ 0 " in Fig. 2b), which can be attributed to the assumed infinitely sharp intensity cutoffs at $\theta_{1}=\alpha$ and $\theta_{2}=\beta$. In practice, those cutoffs are broadened by scattering on the edges of beam-limiting apertures, and the broadening can be introduced simply as

$$
\mathrm{I}_{0} \propto \alpha^{2}+\beta^{2}+\delta^{2}-\sqrt{\left(\alpha^{2}+\beta^{2}+\delta^{2}\right)^{2}-4 \alpha^{2} \beta^{2}}
$$

where $\delta$ is characteristic angle of this "parasitic" scattering.

Dividing Eq. (5) by Eq. (6) produces a complex fitting expression, which was applied to the experimental $\lambda(\beta)$ dependence for $\alpha=3.2 \mathrm{mrad}$ (see curve "Eq. $(5 / 6) \delta=0.1$ mrad" in Fig. 2b). A satisfactory agreement with the experiment is observed. Note that previous intensity normalization (Egerton, 1996, p. 284) is equivalent to using Eq. (5) only, and it results in large deviation from the experiment [see curve "Eq. (2)" in Fig. 2b].

For small convergence semiangles $\alpha$, the fitting equation simplifies into a formula

$$
\frac{1}{\lambda} \propto \frac{\sigma_{\text {in }}}{\mathrm{I}_{0}} \propto \ln \frac{1+\beta^{2} / \theta_{\mathrm{E}}^{2}}{1+\beta^{2} / \theta_{\mathrm{C}}^{2}}
$$


which finally suggests us a compact approximation for $\sigma_{\text {in }} / I_{0}$ for finite $\alpha$ angles as

$$
\frac{1}{\lambda} \propto \frac{\sigma_{\text {in }}}{I_{0}} \propto \ln \left\{\frac{\alpha^{2}+\beta^{2}+2 \theta_{\mathrm{E}}^{2}+\left|\alpha^{2}-\beta^{2}\right|}{\alpha^{2}+\beta^{2}+2 \theta_{\mathrm{C}}^{2}+\left|\alpha^{2}-\beta^{2}\right|} \times \frac{\theta_{0}^{2}}{\theta_{\mathrm{E}}^{2}}\right\}
$$

As a function of $\beta$, Eq. (8) is constant for $\beta<\alpha$ and is equal to Eq. (7) for $\beta>\alpha$. It fits rather well to the experiment [see curve "Eq. (8)" in Fig. 2b] and therefore has been used for fitting the data of Figure $2 \mathrm{a}$.

In all those simulations, the saturation factor $\theta_{\mathrm{C}}$ has been set to $20 \mathrm{mrad}$. The physics behind this constant has been discussed in detail (Egerton, 1996, p. 161) in terms of a critical wavevector of plasmon scattering. This parameter has a complex material dependence via the structure of the Fermi surface. In practice of thickness measurements, this (weak logarithmic) dependence is swamped by the experimental error, and thus $\theta_{\mathrm{C}}$ can well be assumed constant.

\section{CONCLUSIONS}

In this article, we have critically reanalyzed thickness measurements using the well-known log-ratio and Kramers-Kronig sum models of EELS.. Our experimental results do confirm the previously established validity and accuracy of the KK and relative log-ratio models (Egerton and Chen, 1987; Yang and Egerton, 1995) in all respects except for the angular dependence. This dependence versus the convergence and collection angles has been measured and successfully simulated with a simple theory resulting in a compact Eq. (8). Experiment reveals that this equation is a better alternative to the angular term $\ln (1+$ $\beta^{2} / \theta_{\mathrm{E}}^{2}$ ) widely adopted in most theories of inelastic scattering.

The easiest way of thickness measurements with EELS relies on the absolute log-ratio method, which however requires knowledge of the mean free path of inelastic electron scattering $\lambda$. The latter has been measured here in a wide range of solids and a scaling law $\lambda \sim \rho^{-0.3}$ versus mass density $\rho$ has been revealed. The origin of this exponent is currently being investigated. Furthermore, an updated formula for $\lambda$ is proposed as

$$
\begin{gathered}
\frac{1}{\lambda}=\frac{11 \rho^{0.3}}{200 \mathrm{FE}_{0}} \ln \left\{\frac{\alpha^{2}+\beta^{2}+2 \theta_{\mathrm{E}}^{2}+\left|\alpha^{2}-\beta^{2}\right|}{\alpha^{2}+\beta^{2}+2 \theta_{\mathrm{C}}^{2}+\left|\alpha^{2}-\beta^{2}\right|} \times \frac{\theta_{\mathrm{C}}^{2}}{\theta_{\mathrm{E}}^{2}}\right\}, \\
\mathrm{F}=\frac{\left(1+E_{0} / 1022\right)}{\left(1+\mathrm{E}_{0} / 511\right)^{2}}, \quad \theta_{\mathrm{E}}=\frac{5.5 \rho^{0.3}}{\mathrm{FE}_{0}}, \quad \theta_{\mathrm{c}}=20 \mathrm{mrad}
\end{gathered}
$$

where dimensions are $E_{0}(\mathrm{kV})$, all angles (mrad), and density $\left(\mathrm{g} / \mathrm{cm}^{3}\right)$.

\section{ACKNOWLEDGMENTS}

The authors are grateful to $H$. Shima and $H$. Akinaga (AIST, Tsukuba, Japan) for provision of the $\mathrm{Si} / \mathrm{aSiO}_{2} /$ $\mathrm{Ta} / \mathrm{CoO} / \mathrm{Pt} / \mathrm{aC}$ and $\mathrm{Si} / \mathrm{aSiO}_{2} / \mathrm{Ta} / \mathrm{Pt} / \mathrm{NiO} / \mathrm{Al}$ materials. This research was partially supported by Ministry of Education, Science, Sports and Culture (MEXT) through a Grant-in-Aid for Young Scientists (B) 2005 17710120-6816 and the Nuclear Research Project.

\section{REFERENCES}

Crozier PA. 1990. Measurement of inelastic electron scattering crosssections by electron energy-loss spectroscopy. Phil Mag B 61:311-336.

Egerton RF. 1996. Electron energy loss spectroscopy in the electron microscope, 2nd ed. New York: Plenum.

Egerton RF, Cheng SC. 1987. Measurement of local thickness by electron energy-loss spectroscopy. Ultramicroscopy 21:231-244.

Iakoubovskii K, Mitsuishi K, Nakayama Y, Furuya K. 2008. Mean free path of inelastic electron scattering in elemental solids and oxides using transmission electron microscopy: Atomic number dependent oscillatory behavior. Phys Rev B 77:104102.

Jin Q. 2004. Thickness measurements of a TEM foil and its surface layer by electron energy-loss spectroscopy. Microsc Microanal 10:882-883.

Krivanek OL, Ahn CC, Wood GJ. 1990.. The inelastic contribution to high resolution images of defects. Ultramicroscopy 33:177-185.

Lee CW, Ikematsu Y, Shindo D. 2002. Measurement of mean free path for inelastic electron scattering of $\mathrm{Si}$ and $\mathrm{SiO}_{2}$. J Electron Microsc 51:143-148.

Malis T, Cheng SC, Egerton RF. 1988. EELS log-ratio technique for specimen-thickness measurement in the TEM. J Electron Microsc Tech 8:193-200.

Mitchell DRG. 2006. Determination of mean free path for energy loss and surface oxide film thickness using convergent beam electron diffraction and thickness mapping: A case study using Si and P91 steel. J Microsc 224:187-196.

Nakafuji A, Murakami Y, Shindo D. 2001. Effect of diffraction condition on mean free path determination by EELS. J Electron Microsc 50:23-28.

Ohshima K, Kaneko K, Fujita T, Horita Z. 2004. Determination of absolute thickness and mean free path of thin foil specimen by zeta-factor method. J Electron Microsc 53:137-142.

Yang YY, Egerton RF. 1995. Tests of two alternative methods for measuring specimen thickness in a transmission electron microscope. Micron 26:1-5. 